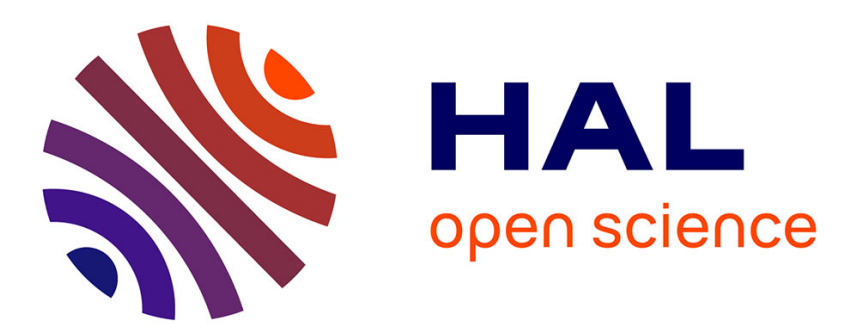

\title{
Un nouveau principe général d'extraction dans les sources d'ions. Applications à différents types de sources. Deuxième partie: résultats d'expériences
}

\author{
J. Sommeria
}

\section{- To cite this version:}

J. Sommeria. Un nouveau principe général d'extraction dans les sources d'ions. Applications à différents types de sources. Deuxième partie: résultats d'expériences. J. Phys. Radium, 1952, 13 (12), pp.651-657. 10.1051/jphysrad:019520013012065100 . jpa-00234665

\section{HAL Id: jpa-00234665 https://hal.science/jpa-00234665}

Submitted on 1 Jan 1952

HAL is a multi-disciplinary open access archive for the deposit and dissemination of scientific research documents, whether they are published or not. The documents may come from teaching and research institutions in France or abroad, or from public or private research centers.
L'archive ouverte pluridisciplinaire HAL, est destinée au dépôt et à la diffusion de documents scientifiques de niveau recherche, publiés ou non, émanant des établissements d'enseignement et de recherche français ou étrangers, des laboratoires publics ou privés. 


\author{
UN NOUVEAU PRINGIPE GÉNÉRAL D'EXTRAGTION DANS LES SOURGES D'IONS. \\ APPLIGATIONS A DIFFÉRENTS TYPES DE SOURGES. \\ DEUXIĖME PARTIE : RÉSULTATS D'EXPÉRIENGES. \\ Par J. SOMMERIA. \\ Ingénieur E. S. E. \\ Laboratoire de Synthèse atomique. Ivry (Seine).
}

\begin{abstract}
Sommaire. - Il est décrit différentes réalisations du nouveau principe d'extraction exposé dans la ${ }_{\text {I }}^{\text {re }}$ partie de cet article [18], ainsi que les résultats obtenus. La comparaison des résultats est effectuée pour chaque type de source fonctionnant avec l'ancien ou le nouveau dispositif d'extraction.
\end{abstract}

Nous avons élaboré un programme d'application à différents types de source du principe d'extraction proposé dans le chapître précédent. Une réalisation a été effectuée pour la source du type "Heil-Von Ardenne ». Quelques expériences ont été réalisées sur la source "Finkelstein " et un projet est en cours d'exécution avec ionisation par champ à haute fréquence.

1. Principe de la source "Heil-Von Ardenne". Résultats obtenus par les auteurs. - Nous avions choisi cette source d'ions en vue de la monter sur un tube accélérateur à $600 \mathrm{kV}$ en raison de ses rendements intéressants par rapport aux consommations de gaz et d'énergie électrique mentionnés par ses auteurs, ainsi que pour le faible étalement d'énergie des ions.

On voit sur la figure 7 sa coupe schématique avec les dimensions principales.

L'ouverture de la chambre d'ionisation en forme de fente ou circulaire est relativement grande $\left(50 \mathrm{~mm}^{2}\right.$ ). Le potentiel le long des trajecioires ioniques et électroniques a les formes représentées sur la figure i $e$ (Première partie).

Le Mémoire de Heil indique les courbes du courant d'ions en fonction de la tension d'extraction pour différentes valeurs du champ magnétique. Le courant maximum varie de 500 à $1250 \mu \mathrm{A}$ pour des champs respectifs de 5on à 6 ooo Gauss (courant électronique, $7 \mathrm{~mA}$; tension d'extraction optima, 50o V).

Le rendement au point de vue de la consommation de gaz est indiqué égal à 1 pour 1 oo; mais les pressions correspondantes dans la chambre d'ionisation et dans l'enceinte de l'appareil utilisateur ne sont pas données avec précision.

Remarquons qu'il n'a pas été prévu de cloisonnement entre ces deux enceintes et qu'il est facile, même dans ce cas, d'obtenir un rendement de roo pour Ioo si les pressions sont égales de part et d'autre.

Nous avons été amené dès le début de nos expé- riences à considérer que les travaux de Heil et Von Ardenne ne constituaient que des essais préliminaires d'un principe de source d'ions susceptible d'ailleurs de donner des résultats très intéressants. La suite de cet exposé indique les principaux résultats expérimentaux de la mise au point de cette source en vue de son adaptation à des conditions d'utilisation précises.

2. Installation d'essais. Procédés de mesures. - a. Installation DE vide. - Elle comprend une pompe à palettes, une pompe à vapeur d'huile

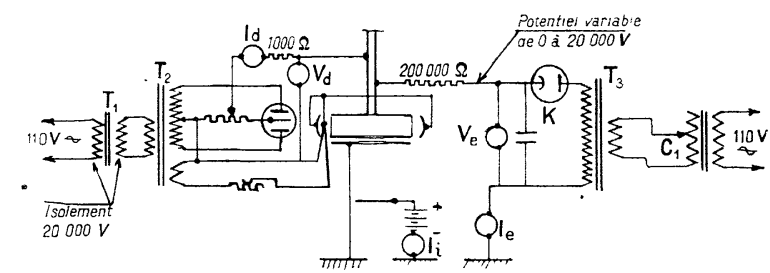

Fig. 5. - Schéma des circuits électriques pour les essais de sources.

de 1 oo $\mathrm{l} / \mathrm{s}$, une vanne de $120 \mathrm{~mm}$ d'ouverture et se termine par un plateau horizontal sur lequel peut être monté une cloche ou un manchon de verre de grand diamètre. Une jauge d'ionisation est placée immédiatement au-dessous du plateau.

$b$. Circuits électrigues D'alimentation et DE MEsures. - La figure $\tilde{J}$ indique tous les détails de l'installation.

c. Montage expérimental pour les sources du type " Heil-Von Ardenne ". - La photographie $\mathrm{n}^{\circ} 1$ en indique une vue d'ensemble.

Les électrodes relatives à la décharge ionisante sont montées sur un socle cylindrique en laiton par l'intermédiaire de supports-traversées en verrecovar isolés pour i 5 ooo $\mathrm{V}$ environ. Le filament en tungstène de $15 / 100 \mathrm{~mm}$ a une longueur de $2 \mathrm{~cm}$. 
L'ensemble est placé dans l'entrefer d'un aimant dont le champ peut se régler en "shuntant " une partie de son circuit magnétique par des pièces de fer. Le champ maximum est de 1500 gauss pour un entrefer de $3 \mathrm{~cm}$ de longueur.

L'électrode d'extraction, toujours reliée à la terre, est montée sur un chapeau qui coiffe l'ensemble de la source et qui peut servir éventuellement à l'isoler du milieu extérieur avec une étanchéité relative. On voit sur la photographie ce chapeau posé sur le socle de l'aimant.

L'extraction des ions se fait vers le haut. L'ensemble de l'aimant et de la source est placé dans la cloche à vide. On peut ainsi observer facilement la forme du faisceau d'ions. Celui-ci est reçu sur une plaque métallique connectée au circuit de mesure.

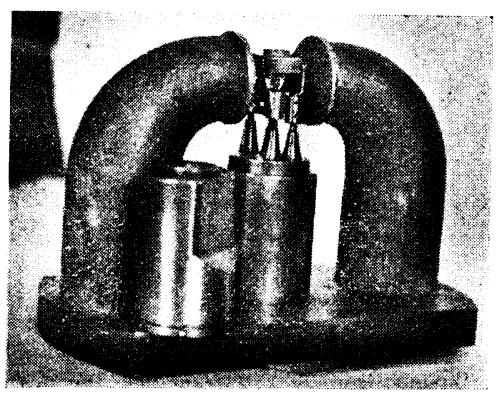

Photo no 1.

Le gaz à ioniser est emmagasiné dans un ballon de verre et le réglage de son débit est effectué à l'aide d'un robinet à aiguille.

d. Procédés de mesures. - Indépendamment des quantités électriques qui sont mesurées avec les appareils représentés sur les schémas, il est indispensable de connaître à chaque instant le débit de gaz, les pressions dans la source et à l'extérieur.

Nous mentionnerons rapidement les procédés de ces mesures sans entrer dans les détails pour ne pas surcharger cet exposé.

La jauge d'ionisation indique avec une bonne précision la pression à l'extérieur de la source, quel que soit le débit de gaz. Elle est, en effet, placée à peu de distance de celle-ci et dans une canalisation de très grand diamètre (ioo $\mathrm{mm}$ ). Nous l'avons étalonnée en atmosphère d'hydrogène à l'aide d'une jauge de Mac Leod. Ses indications sont proportionnelles à la pression dans les limites normales d'utilisation avec un coefficient de $3.10^{-5} \mathrm{~mm} \mathrm{Hg} / \mu \mathrm{A}$ (jauge de la Compagnie de Radiologie; courant ionisant, $20 \mathrm{~mA}$ ). Remarquons que ce coefficient est très différent de ceux donnés généralement pour l'air ou d'autre gaz et que d'importantes erreurs dans les mesures de pression semblent avoir été commises dans certains Mémoires où les auteurs ont probablement utilisé des coefficients moyens.
Les électrodes de la source ont été utilisées pour mesurer la pression dans la chambre d'ionisation. La figure 6 représente le montage effectué comme pour une jauge d'ionisation. Les réflecteurs ont été isolés; la résistance $R$ produit la polarisation nécessaire pour que le réflecteur de droite capte les ions. $I$ est le courant ionisant et $i$ le courant fonction de la pression. Une courbe d'étalonnage à été tracée par comparaison avec la jauge d'ionisation du banc de pompage. L'égalité des pressions dans la source et dans le banc a été obtenue en situant l'arrivée de gaz de manière à laisser la source et la jauge en dehors du circuit de pompage.

La jauge d'ionisation a été également étalonnée en débit de gaz. Les variations de pression au-dessus du vide limite sont proportionnelles au débit de gaz (mesuré, par exemple, en centimètres cube par heure à la pression atmosphérique); elles sont dues, en effet, au passage du gaz dans la résistance du circuit

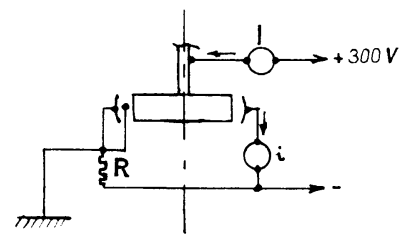

Fig. 6. - Montage de la source en jauge d'ionisation.

de pompage (coefficient de proportionnalité pour notre installation, $11,6 \mathrm{~cm}^{3} / \mathrm{h} . \mu \mathrm{A}$ d'H à la pression atmosphérique).

3. Reproduction de la source "Heil-Von Ardenne originale Mesures. - Une réalisation de la source conforme à la description de ses auteurs était indispensable pour constituer le point de départ de nos expériences.

Avant de réaliser l'aimant de I 500 gauss représenté sur la photographie, nous disposions d'un aimant de 700 gauss qui nous a servi à effectuer les premières mesures.

Malgré l'augmentation du rendement d'ionisation avec le champ magnétique mise en évidence par Heil, nous n'avons pas cru intéressant d'utiliser un champ élevé obligeant à employer un électroaimant et à consommer une énergie électrique importante. Celle-ci devait être, en effet, fournie sur le tube accélérateur par des piles et des accumulateurs.

Nous donnons quelques résultats de mesures effectuées sur la source originale ( $f i g .7)$ pour permettre la comparaison avec le montage définitif muni de la lentille de concentration.

Les courbes de la figure 7 indiquent, d'une part le courant d'ions $I_{i}$ en fonction de la tension d'extraction $\left(V_{e}\right)$ pour une tension de décharge de $450 \mathrm{~V}$ $\left(V_{d}\right)$, un courant électronique de $\mathrm{I}_{4} \mathrm{~mA}$ et une pression de $2.10^{-3} \mathrm{~mm}$ de $\mathrm{Hg}$ et, d'autre part, la 
variation correspondant du courant capté par l'électrode d'extraction $\left(I_{e}\right)$.

De nombreuses courbes ont été tracées pour différentes valeurs de la pression et du courant

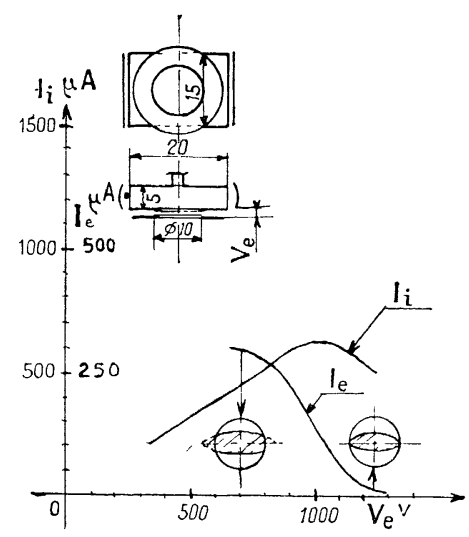

Fig. 7. - Partie supérieure : Deux vues de la source originale de Heil-Von Ardenne.

Partie inférieure : courbes du courant d'ions et du courant absorbé par l'électrode d'extraction en fonction de la tension d'extraction.

Section du faisceau d'ions sur le plan de l'électrode d'extraction correspondant à deux points des courbes.

Champ, 700 gauss; Courant de décharge, $14 \mathrm{~mA}$; Pression, $2.10^{-3} \mathrm{mmHg}$.

électronique; mais pour simplifier et seulement dans un but de comparaison, nous n'indiquons que celles qui correspondent aux caractéristiques de fonctionnement optimum de la source définitive.

On voit que le courant d'ions passe par un maximum pour une certaine tension d'extraction ( $750 \mathrm{~V})$. En effet, le champ extracteur, à partir d'une certaine valeur, a deux actions contradictoires sur le milieu ionisé; il augmente le volume d'action dans celui-ci, mais aussi sa déformation et, par suite, le dérangement du processus d'ionisation.

On voit aussi que le courant capté par l'électrode d'extraction $\left(I_{e}\right)$ diminue très rapidement aux environs du maximum de $I_{i}$, ce qui tend à prouver que le faisceau d'ions est large par rapport à l'ouverture de cette électrode (Io mm).

Ceci se vérifie d'ailleurs visuellement, car le faisceau est suffisamment lumineux dans l'atmosphère résiduelle de la cloche d'observation.

On voit aussi sur la figure 7 la forme de la section de passage des ions sur le plan de l'électrode d'extraction qui a été aussi déterminée par les traces laissées sur des feuilles d'aluminium poli.

La forme de la section du faisceau est indiquée avant et après la chute du courant $I_{e}$.

La concentration augmente légèrement avec la tension d'extraction, mais le faisceau d'ions reste constamment divergent avec un angle considérable à partir de l'ouverture de la chambre d'ionisation.

L'ovalisation du faisceau est une aberration due au champ magnétique. Ceci peut s'expliquer par l'effet de celui-ci sur les ions de faible vitesse, au début de leur extraction. Il se produit alors une dispersion de vitesse qui semble symétrique par rapport à l'axe du faisceau. Cet effet est indépendant d'une déflexion générale de celui-ci.

On voit que l'utilisation de cette source avec le dispositif d'extraction original conduirait à l'emploi d'un diaphragme de grandes dimensions et, par suite, à un très mauvais rendement par rapport à la consommation de gaz. Une optique ionique convenable de l'appareil utilisateur serait aussi très difficile à réaliser avec un "objet" de si grandes dimensions.

\section{Nouvelles sources à grande concentration} d'ions. - On voit sur la figure 8 une coupe schématique de la chambre d'ionisation et du dispositif de concentration tel qu'il a été décrit dans le chapitre précédent.

L'électrode d'extraction est complètement ouverte pour permettre la mesure du courant total d'ions et pour déterminer ensuite la perte de courant dans la traversée du diaphragme. La courbe correspondante montre la variation du courant d'ions en fonction de la tension d'extration et de concentration pour une pression de $2 . \mathrm{I}^{-3} \mathrm{~mm} \mathrm{Hg} \mathrm{d} H$ et un courant de décharge de $14 \mathrm{~mA}$. Le maximum d'intensité $(\mathrm{I}, \mathrm{I} 5 \mathrm{~mA})$ est atteint pour $V_{g}=\mathrm{I} 5000 \mathrm{~V}$.

La position et la forme du diaphragme sont déterminées à l'aide de la trace du faisceau sur une pièce

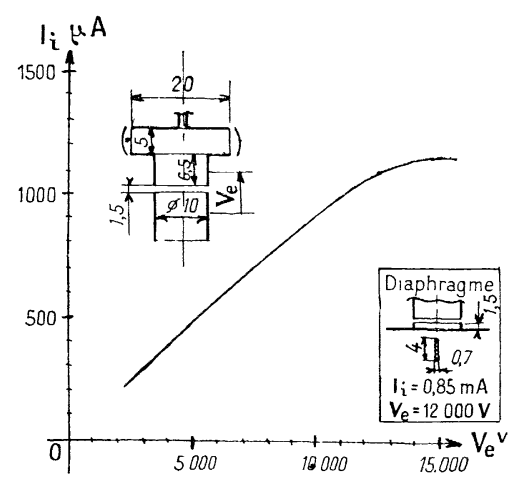

Fig. 8. - Courbe du courant d'ions en fonction de la tension d'extraction pour la disposition représentée à gauche.

Champ magnétique, 700 gauss; Courant de décharge, $14 \mathrm{~mA}$; Pression : $2.10^{-3} \mathrm{mmHg}$.

Dans le cadre de droite: Le diaphragme tracé et percé et les résultats obtenus pour les mêmes autres conditions.

de cuivre polie électrolytiquement et ajustée dans l'électrode d'extraction. Le perçage s'effectue d'après la trace obtenue.

La figure 8 représente également la position et la forme du diaphragme pour les caractéristiques de fonctionnement précédentes (fente très fine de $2,8 \mathrm{~mm}^{2}$ de surface). On retrouve ici le phénomène 
d'aberration dans une direction perpendiculaire au champ magnétique.

Remarquons que la forme en lame du faisceau est relativement favorable au point de vue de l'effet de sa charge d'espace.

L'aberration unidirectionnelle particulière à cette source laisse la possibilité de réaliser un diaphragme dont une des dimensions et, par suite, la section, est très petite. Ceci constitue un avantage important par rapport aux sources à champ magnétique longitudinal pour lesquelles on ne peut pas s'affranchir de l'aberration qui a lieu dans toutes les directions (voir première partie, chap. II, $\S b$ ).

Le courant traversant le diaphragme pour les conditions de fonctionnement de la source réalisées pendant son tracé est de $0,85 \mathrm{~mA}$ (pour $V_{s}=12000 \mathrm{~V}$ $I_{e}=14 \mathrm{~mA}$; pression $2.10^{-3} \mathrm{~mm} \mathrm{Hg}, H=700$ gauss,

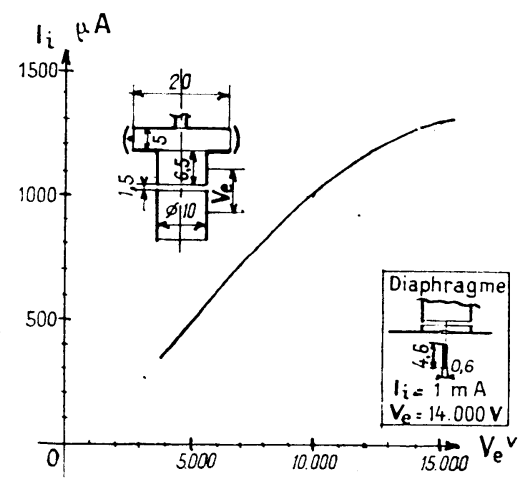

Fig. 9. - Courbe du courant d'ions en fonction de la tension d'extraction pour la disposition représentée à gauche.

Champ magnétique, 1500 gauss; Courant de décharge, $14 \mathrm{~mA}$; Pression d'hydrogène, 2. $10^{-3} \mathrm{mmHg}$.

Dans le cadre de droite : Le diaphragme tracé et percé et les résultats obtenus pour les mêmes autres conditions.

alors qu'il était au maximum de 1, $55 \mathrm{~mA}$ avec l'électrode d'extraction complètement ouverte. La tension correspondant au maximum est plus petite avec le diaphragme qu'avec l'électrode ouverte. La différence correspond, en effet, aux surfaces équipotentielles supprimées par la présence de la paroi du diaphragme. Nous l'avons déterminée d'après la carte des équipotentielles tracées à la cuve rhéographique.

La consommation d'hydrogène correspondant à ce fonctionnement est de $9 \mathrm{~cm}^{3} / \mathrm{h}$ à la pression atmosphérique, ce qui donne un rendement de 8,5 pour ioo.

Avec le même montage, nous avons utilisé un champ magnétique de i 500 gauss. La courbe de la figure 9 montre les nouveaux résultats obtenus (électrode d'extraction ouverte).

Un nouveau tracé et un nouveau perçage du diaphragme ont été réalisés avec les résultats suivants :

Courant d'ions, I $\mathrm{mA}$;
Tension d'extraction et de concentration $14000 \mathrm{~V}$; Courant de décharge, $14 \mathrm{~mA}$;

Pression d'hydrogène, $2.10^{-3} \mathrm{~mm} \mathrm{Hg}$;

Diaphragme, 2,8 $\mathrm{mm}^{2}$;

Consommation de gaz, $9 \mathrm{~cm}^{3} / \mathrm{h}$;

Rendement par rapport au gaz, io pour ıoo;

Puissance électrique absorbée, ı $3 \mathrm{~W}$ (décharge, 4 W; filament, $9 \mathrm{~W}$ );

Rendements électriques $77 \mu \mathrm{A} / \mathrm{W}$ et 7 pour 100 .
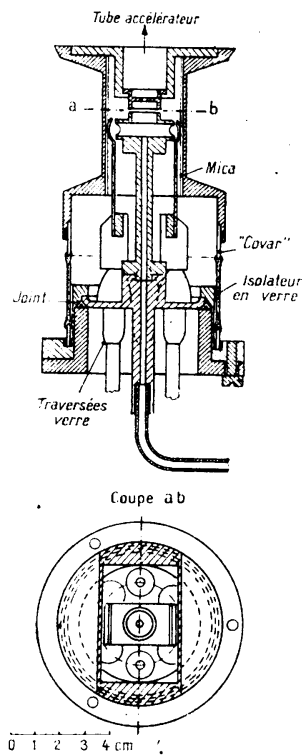

Fig. 1 o. - Source définitive avec aimant extérieur.

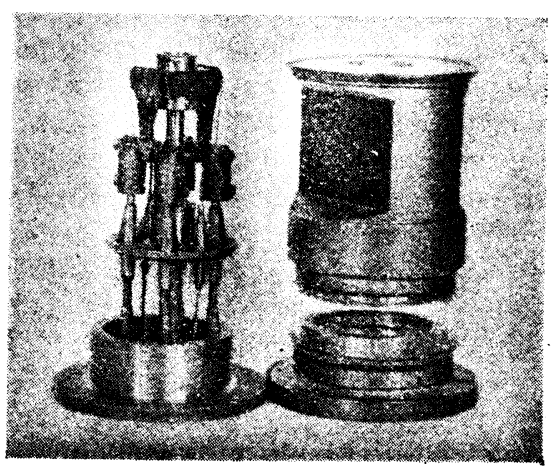

Photo no 2.

Le dessin de la figure 10 et la photographie $\mathrm{n}^{0} 2$ montrent la source d'ions définitive réalisée avec l'aimant extérieur. On voit sur la partie droite de la photographie l'enceinte de la source portant l'électrode d'extraction. On aperçoit l'isolateur en verre soudé au métal qui supporte la tension de 15 ooo V.

La photographie no 3 montre la source en fonctionnement avec le faisceau d'ions visible dans l'enceinte à vide dont on peut remarquer la grande 
finesse. L'ensemble de l'enceinte est réuni à la terre. On aperçoit la plaque collectrice des ions.

Les courants d'ions indiqués dans tout ce qui précède comprennent l'ensemble des molécules et

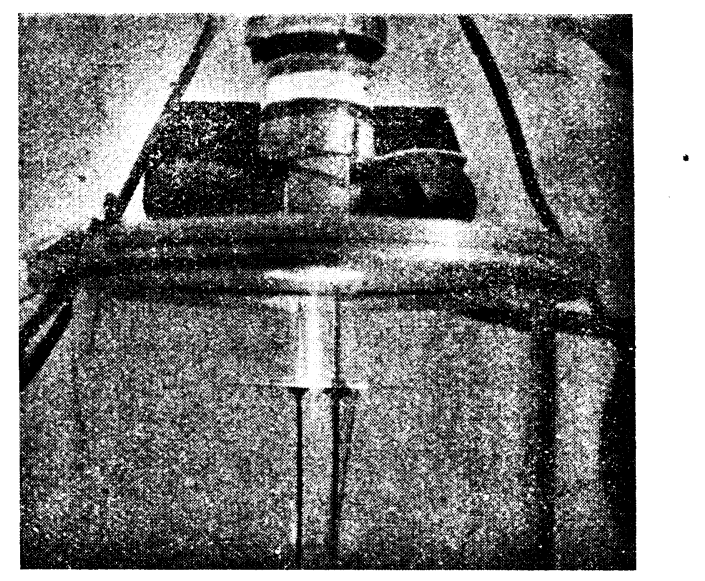

Photo no 3.

des atomes ionisés. Des mesures spectrographiques pour l'analyse $\mathrm{du}$ faisceau d'ions sont réalisées actuellement et feront l'objet de publications ultérieures.

Le rendement en atomes dépend principalement du système d'ionisation, mais le procédé d'extraction intervient aussi certainement. Cètte étude n'entre pas dans le cadre de cet exposé qui a pour seul but de démontrer expérimentalement l'efficacité du nouveau principe d'extraction.

5. Application du nouveau principe d'extraction à une source du type "Finkelstein ». Quelques essais préliminaires. - La figure 12 montre quelques applications de notre principe d'extraction à " lentille cathodique " sur différents types de source, et permet la comparaison avec les systèmes classiques représentés sur la figure I (Première partie).

La figure i $2 \boldsymbol{d}$ montre la disposition relative à la source "Finkelstein ". Le schéma est le même que pour le type classique, mais la tension entre $a$ et $c$ est bien supérieure à celle entre $a$ et $b$. La courbe de droite indique la forme du potentiel sur l'axe. Les électrons partant du filament $\mathrm{F}$ sont d'abord accélérés par l'électrode $a$ et "rebondissent " ensuite sur une surface équipotentielle du champ situé entre $a$ et $c$. La chambre d'ionisation n'est donc qu'une partie du cylindre $a$; l'autre partie forme la " lentille cathodique " de concentration des ions.

Ce montage présente l'inconvénient suivant :

La petite fraction du faisceau d'ions qui rencontre toujours le bord du diaphragme produit un courant d'électrons secondaire important qui est accéléré dans le champ de concentration et arrive à provoquer un phénomène de décharge.

La figure 1 I indique schématiquement la disposition et les dimensions des électrodes de la source qui a donné les premiers résultats intéressants, ainsi que la forme approximative du potentiel sur l'axe.

La chambre d'ionisation a été diaphragmée du côté du filament pour réduire l'espace de ${ }_{\mathrm{I}}^{\mathrm{re}}$ accélération des électrons par rapport à celui du $\mathrm{i}$ er ralentissement. Les ions formés dans le ${ }_{\mathrm{I}}{ }^{\mathrm{er}}$ espace sont en effet captés par le filament, et l'on a intérêt à le réduire le plus possible par rapport à l'espace utile d'ionisation.

Une électrode supplémentaire a été ajoutée, pour arrêter les électrons secondaires.

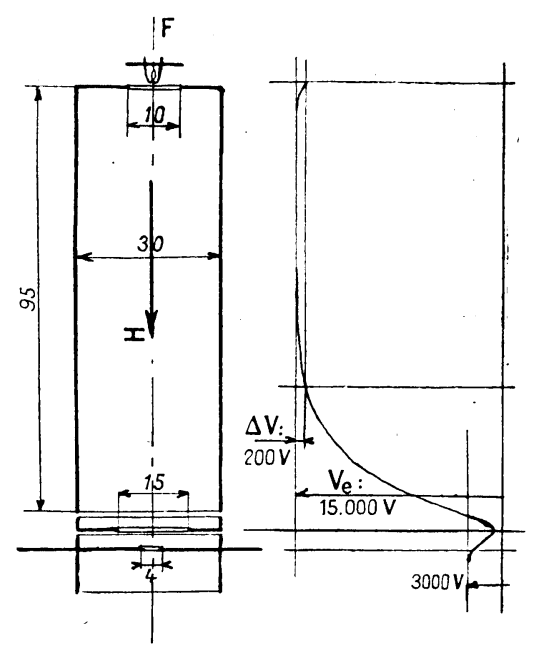

Fig. I r. - Source du type "Finkelstein " à extraction par " lentille cathodique " avec électrode d'arrêt des électrons secondaires.

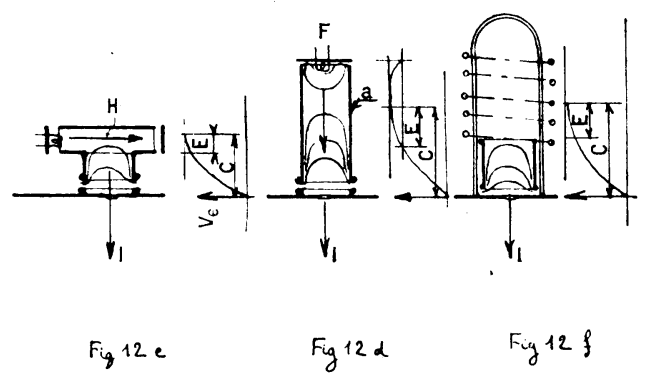

Fig. 12. - Application de la "lentille cathodique " à trois types de sources.

Les indications correspondent à celles de la figure $\mathrm{I}$.

Pésultats obtenus :

Courant ionique total, I,5 mA;

Courant de décharge, $5 \mathrm{~mA}$;

Tension de décharge, $200 \mathrm{~V}$;

Tension d'extraction et de concentration, 15 ooo V;

Diaphragme, $\varnothing 4 \mathrm{~mm}$; 
Pression, $0,7 \cdot \mathrm{IO}^{-3} \mathrm{~mm} \mathrm{Hg}$;

Champ magnétique, I 200 gauss;

Rendement gazeux, io pour гоo;

Rendement électrique $\left(\frac{\text { courant ionique }}{\text { courant de décharge }}\right)$, 3o pour ioo.

Remarques. - La principale caractéristique de cette source est son très bon rendement électrique.

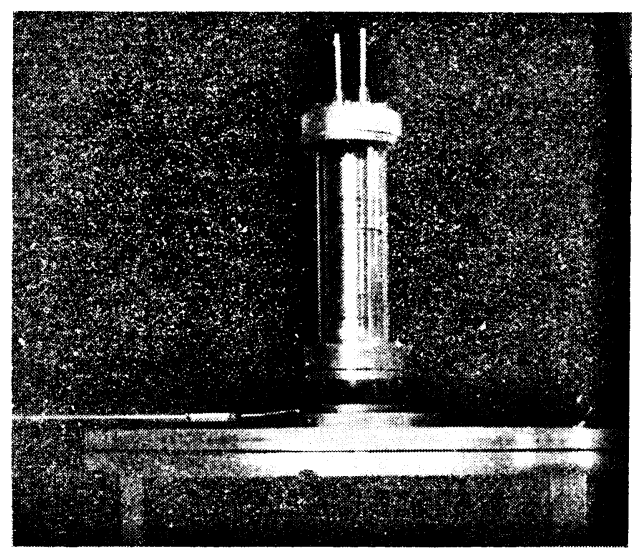

Photo no 4.

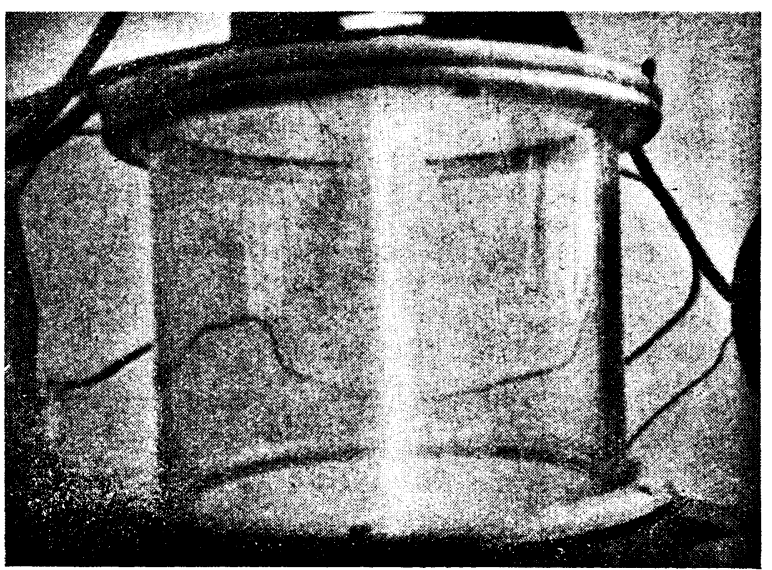

Photo no 5.

La concentration des ions pour une même tension est moins bonne que dans la source précédente, par suite de l'aberration magnétique qui a lieu dans toutes les directions (voir première partie, chap. II, * $1, b)$.

La photographie no 4 montre une vue d'ensemble de la source. L'isolement des électrodes est obtenu par des manchons de verre.

La photographie no 5 montre le faisceau d'ions sortant de la source en fonctionnement.
6. Application à une source à haute fréquence. - La figure i $f$ montre l'application de la " lentille cathodique " à la source à haute fréquence Le solénoïde d'excitation doit être placé de manière à produire la décharge juste au-dessus du cylindre anodique $a$. La courbe du potentiel sur l'axe montre les zones d'extraction et de concentration des ions

Ce principe d'extraction a été à peu près réalisé par A. G. Ward [13]. Il semble cependant que le montage pratique n'ait pas été réalisé pour profiter au maximum des avantages de la " lentille cathodique ". Le cylindre anodique $a$ a en effet une très petite section $\left(\mathrm{I} \mathrm{cm}^{2}\right)$ par rapport à celle de la décharge ( I oo $\left.\mathrm{cm}^{2}\right)$.

Nous avons construit une source avec un cylindre anodique de grande section par rapport à celle de la décharge; mais ces essais n'ont pu encore commencer, car la mise au point du générateur à haute fréquence n’est pas terminée.

7. Comparaison des différents types de source. Conclusion. - La plupart des auteurs dressent à la fin de leurs Mémoires un tableau permettant la comparaison rapide des caractéristiques des différents types de source et notamment de leurs rendements.

La lecture rapide de ces tableaux peut conduire à des erreurs d'appréciation, car les différentes caractéristiques d'une source ne sont pas toujours indiquées pour des mêmes conditions de fonctionnement. Par exemple, les rendements gazeux et électriques varient toujours en sens inverse en fonction du courant de décharge ou du courant d'ions extrait, et les auteurs ont tendance à les indiquer pour deux valeurs de ce dernier qui les rendent les plus favorables.

A la place d'un de ces tableaux, nous dirons quelques mots sur les Mémoires relatifs aux types de source qui semblent actuellement les plus intéressants et sur lesquels il est possible d'utiliser notre principe d'extraction.

a. Sources du type "Heil-Von Ardenne ».-Leur utilisation avait été pratiquement abandonnée étant donné les difficultés particulières présentées pour la concentration des ions.

Nous avons vu que le rendement gazeux indiqué par ses auteurs était de I pour ıoo pour un champ magnétique de 6 ooo gauss.

Avec notre dispositif d'extraction, nous avons obtenu io pour ioo pour un champ magnétique de 1500 gauss seulement.

Notre rendement électrique de 7 pour 100 correspondant à ce même rendement gazeux peut être augmenté en utilisant un champ magnétique plus intense. En général, les performances de cette source, notamment au point de vue du courant d'ions, pourraient être améliorées, mais nous nous étions fixé jusqu'à présent le but de $\mathrm{I} \mathrm{mA}$ suffisant pour un tube accélérateur à haute tension. 
$b$. Sources du trpe "Finkelstein ". - Les sources décrites par Lorrain [5], Veenstra et Milatz [6] Kistmaker et Douwes Dekker [7] représentent les réalisations les plus récentes du type "Finkelstein ».

Les rendements n'ont pas toujours été indiqués, mais nous les avons calculés approximativement, d'après les données des auteurs.

Lorrain, qui utilise une cathode froide, indique un rendement électrique de 1,3 pour ıoo pour une tension de décharge de $300 \mathrm{~V}$, ce rendement augmentant avec la tension. La tension de décharge joue, en effet, dans cette source le même rôle que notre tension de concentration; mais sa valeur trop élevée compromet la finesse du spectre d'énergie. Le rendement gazeux est de 6 pour 1 oo environ. Veenstra et Milatz utilisent une cathode chaude. Nous avons calculé un rendement électrique de 2 pour ioo et un rendement gazeux de i 2 pour ıoo environ.

Kistmaker et Douwes Dekker ont réalisé un dispositif de concentration des ions original en plaçant la cathode chaude entre le cylindre anodique et le diaphragme d'extraction. Celle-ci, qui est constituée par un filament de tungstène enroulé en anneau, réalise une zone à grande densité de charge négative qui compense la charge positive du faisceau d'ions. Le rendement gazeux indiqué de 5o pour 100 paraît considérable, mais il n'est pas précisé s'il correspond au fonctionnement en hélium, azote ou hydrogène, ces trois gaz étant étudiés par les auteurs. Si le courant d'ions n'est pas limité par sa. charge d'espace, il est plus facile d'obtenir un bon rendement gazeux avec un gaz lourd, car sa vitesse d'écoulement à travers le diaphragme est plus petite. (La vitesse d'écoulement est inversement proportionnelle à la racine carrée de la masse moléculaire.) La vitesse indiquée dans le Mémoire ( $12 \mathrm{l} / \mathrm{s} . \mathrm{cm}^{2}$ ) correspond à peu près à l'azote; pour l'hydrogène, elle est environ quatre fois plus grande.

Le rendement électrique est de 4 pour ıoo.

Nous avons vu que les rendements gazeux et électrique de notre source du type "Finkesitein " étaient respectivement de so et 3 o pour ioo pour l'hydrogène.
Nous pouvons donc constater que pour ce type de source, notre dispositif de concentration n'a amélioré jusqu'à présent que le rendement électrique (de 4 à 3 o pour ıoo). Ceci prouve, comme nous l'avions prévu, que l'extraction agit sur une grande partie du volume ionisé, mais le processus de concentration est gêné considérablement par l'aberration magnétique due au champ longitudinal.

c. Sources a haute fréquence. - Les deux premières sources de ce type mises au point par Thonemann et A. G. Ward donnent un rendement gazeux de 3 pour roo environ (50o $\mu \mathrm{A}$ d'ions, I $5 \mathrm{~cm}^{3} / \mathrm{h}$ de consommation d'hydrogène). Le rendement électrique est très faible $(2 \mu \mathrm{A} / \mathrm{W})$.

Les derniers travaux de C. D. Moak, H. Reese et Good [13] consistent en une source de "Thonnemann " alimentée en très grande puissance (5ooW). Le rendement gazeux atteint ainsi une très bonne valeur (ı9 pour ıоo pour $1,2 \tilde{\mathrm{mA}}$ d'ions), mais le rendement électrique demeure très faible $(2,5 \mu \mathrm{A} / \mathrm{W}$.)

Nous pensons pouvoir améliorer considérablement ce dernier rendement par l'utilisation de notre principe d'extraction dont les essais vont commencer incessamment. Son efficacité sera certainement très grande dans ce cas où l'aberration magnétique n'existe pas.

d. Source annulaire. - Nous avons proposé dans une "Lettre à l'éditeur " [17] une source à extraction par lentille cathodique annulaire.

Nous pouvons conclure qu'avec tous les dispositifs d'ionisation sans champ magnétique constant ou avec un champ magnétique transversal n'introduisant qu'une aberration unidirectionnelle, le système de concentration des ions à " lentille cathodique" peut constituer une amélioration importante par rapport à la méthode classique

Nous tenons à remercier le Directeur du Laboratoire de Synthèse atomique, M. le Professeur F. Joliot-Curie, pour l'intérêt qu'il a apporté à ce travail.

Manuscrit reçu le is juillet ${ }_{1952}$.

\section{BIBLIOGRAPHIE.}

De ]1] à [16 ], voir bibliographie de la première partie.
[17] Sommeria J. - J. Physique Rad., i 95 I, 12, 563. [18] Sommeria J. - J. Physique Rad., i $952,13,645$. 